\title{
Relationship between PTEN, DNA mismatch repair, and tumor histotype in endometrial carcinoma: retained positive expression of PTEN preferentially identifies sporadic non-endometrioid carcinomas
}

\author{
Bojana Djordjevic ${ }^{1,3}$, Bedia A Barkoh², Rajyalakshmi Luthra ${ }^{2}$ and Russell R Broaddus ${ }^{1}$ \\ ${ }^{1}$ Department of Pathology, University of Texas M.D. Anderson Cancer Center, Houston, TX, USA and \\ ${ }^{2}$ Department of Hematopathology, University of Texas M.D. Anderson Cancer Center, Houston, TX, USA
}

\begin{abstract}
Loss of PTEN (phosphatase and tensin homolog) expression and microsatellite instability are two of the more common molecular alterations in endometrial carcinoma. From the published literature, it is controversial as to whether there is a relationship between these different molecular mechanisms. Therefore, a cohort of 187 pure endometrioid and non-endometrioid endometrial carcinomas, carefully characterized as to clinical and pathological features, was examined for PTEN sequence abnormalities and the immunohistochemical expression of PTEN and the DNA mismatch repair proteins MLH1, MSH2, MSH6, and PMS2. MLH1 methylation analysis was performed when tumors had loss of MLH1 protein. Mismatch repair protein loss was more frequent in endometrioid carcinomas compared with non-endometrioid carcinomas, a difference primarily attributable to the presence of MLH1 methylation in a greater proportion of endometrioid tumors. Among the nonendometrioid group, mixed endometrioid/non-endometrioid carcinomas were the histotype that most commonly had loss of a mismatch repair protein. In endometrioid tumors, the frequency of PTEN loss measured by immunohistochemistry and mutation did not differ significantly between the mismatch repair protein intact or mismatch repair protein loss groups, suggesting that PTEN loss is independent of mismatch protein repair status in this group. However, in non-endometrioid carcinomas, both intact positive PTEN immunohistochemical expression and PTEN wild type were highly associated with retained positive expression of mismatch repair proteins in the tumor. Relevant to screening endometrial cancers for Lynch Syndrome, an initial PTEN immunohistochemistry determination may be able to replace the use of four mismatch repair immunohistochemical markers in $63 \%$ of patients with non-endometrioid endometrial carcinoma. Therefore, PTEN immunohistochemistry, in combination with tumor histotype, is a useful adjunct in the clinical evaluation of endometrial carcinomas for Lynch Syndrome.
\end{abstract}

Modern Pathology (2013) 26, 1401-1412; doi:10.1038/modpathol.2013.67; published online 19 April 2013

Keywords: DNA mismatch repair; endometrial cancer; PTEN

Correspondence: Dr RR Broaddus, Department of Pathology, Unit 85, University of Texas M. D. Anderson Cancer Center, 1515 Holcombe Boulevard, Houston, TX 77030, USA.

E-mail: rbroaddus@mdanderson.org

${ }^{3}$ Current address: Department of Pathology and Laboratory Medicine, University of Ottawa, The Ottawa Hospital, Ottawa, ON, Canada

This original research was presented, in part, at the 99th United States and Canadian Academy of Pathology Annual Meeting, March 20-26, 2010, Washington, D.C., USA.

Received 2 July 2012; revised 18 February 2013; accepted 21 February 2013; published online 19 April 2013
Microstatellite instability (MSI) and loss of phosphatase tensin analog (PTEN) protein function are two of the more common molecular alterations in endometrial carcinoma. In Hereditary Non-Polyposis Colorectal Cancer Syndrome (Lynch Syndrome), which is associated with $1.8-6 \%$ of all endometrial cancers, ${ }^{1,2}$ high levels of microsatellite instability (MSI-high) result from germline mutations of DNA mismatch repair genes MLH1, MSH2, MSH6, and PMS2. MSI-high is also detected in 15-20\% of sporadic endometrial carcinomas ${ }^{3,4}$ due to methylation and subsequent transcriptional silencing of the MLH1 gene promoter. The PTEN 
protein acts as a negative regulator of the PI3K-AKT pathway. PTEN mutations have been found in as many as $34-55 \%$ of endometrial cancers. ${ }^{5,6}$ Studies of PTEN in endometrial carcinoma have typically focused on PTEN sequence abnormalities as determined by sequencing. However, functional PTEN loss in endometrial carcinoma can be mediated by a number of other mechanisms, including PTEN gene promoter methylation, regulation of the PTEN gene or PTEN pseudogene by microRNAs, or alterations of PTEN protein stability and degradation mechanisms. ${ }^{7}$ We have recently demonstrated that PTEN immunohistochemistry identifies $89 \%$ of cases with PTEN sequence abnormality, while also detecting PTEN protein loss in $44 \%$ of cases classified as PTEN wild type by gene sequencing. Therefore, PTEN immunohistochemistry more accurately detects endometrial tumors with functional PTEN loss. ${ }^{8}$

The relationship between PTEN loss and MSIhigh has been a subject of some debate in the published literature. Both PTEN loss ${ }^{9-11}$ and MSIhigh ${ }^{12,13}$ have been identified in complex endometrial hyperplasia, a precursor of endometrial carcinoma, and are regarded as early events in the pathogenesis of endometrioid-type endometrial cancer. ${ }^{5,6}$ It has been hypothesized that the polyadenosine tracts in the PTEN gene make it a target for mutation in the setting of MSI-high. ${ }^{6}$ Several investigators have reported that many PTEN mutations associated with MSI-high tend to occur with greater frequency in these regions compared with PTEN mutations found in microsatellite stable (MSS) tumors. ${ }^{6,14-19}$ On the other hand, one group reported no difference in the spectrum of PTEN mutations between the MSI-high and MSS groups, ${ }^{14}$ while in another study, a large portion of PTEN mutations in MSI-high tumors involved deletions of $\geq 3$ base pairs, sequence abnormalities unlikely to result from MMR protein dysfunction. One explanation for these disparate findings is that MSI-high may not be necessary for the development of all PTEN mutations. Indeed, two studies reported MSI-high and PTEN mutations in endometrial endometrioid adenocarcinoma and only PTEN mutations in complex endometrial hyperplasia, supporting this suggestion. ${ }^{11,15}$

It is also not clear whether relative frequencies of PTEN loss and MSI-high differ in endometrial tumors depending on tumor histotype. Several studies have shown that MSI-high endometrioid endometrial carcinomas are associated with a greater frequency of PTEN mutations compared with MSS tumors. ${ }^{6,14-19}$ In all of these studies, MSI was assessed using PCR. Two studies ${ }^{18,20}$ have shown a correlation between immunohistochemical PTEN loss and PCR-based MSI-high. In one study, within a cohort of endometrioid and non-endometrioid carcinomas, PTEN loss was observed in 40\% (4/10) of MSI-high cases compared with $11 \%$ (9/79) of MSS cases. Of the 10 MSI-high cases, all were of endometrioid histology. ${ }^{18}$ Another study investigated predominantly endometrioid tumors, finding either a decrease or loss of PTEN expression in $76 \%(13 / 17)$ of MSI-high cases compared with $49 \%(37 / 76)$ of MSS cases. MSI-high was not detected in any of the 7 non-endometrioid tumors examined. ${ }^{20}$

In the past publications, PTEN loss in relation to MSI-high in non-endometrioid tumors has been studied only to a limited extent, focusing particularly on serous and clear-cell carcinomas. PTEN mutations have been found in $0-14 \% 14,15,19,21,22$ and $5-18 \%{ }^{21,23}$ of these tumors, respectively, while MSIhigh has been found in $0-28 \% 14,20,24-26$ of serous and in $0-9 \%$ of clear-cell carcinomas. ${ }^{20,26}$ In addition, many of these studies were conducted on small case cohorts and/or with non-endometrioid tumors representing $<25 \%$ of the tumors examined.14,15,19,22,25,27 PTEN mutations and/or PTEN immunohistochemical protein loss may not be restricted to endometrioid tumors, as these changes can also be detected in other histotypes, such as mixed endometrioid/non-endometrioid endometrial carcinomas (31\%), undifferentiated carcinomas $(60 \%)$, and carcinosarcomas $(8 \%){ }^{8}$

In order to make screening of endometrial carcinoma patients for Lynch Syndrome feasible, the screening tools must be easily accessible, reliable, and incur minimal cost. The discussion above suggests that PTEN loss, either assessed by sequencing or immunohistochemistry, may be a useful addition to immunohistochemistry, MSI analysis, and MLH1 methylation analysis in the clinical workup of Lynch syndrome. The central objective of this study was to determine how tumor histotype in combination with PTEN status correlates with MMR protein loss, and whether this information can help triage endometrial cancer patients who require additional clinical testing for Lynch Syndrome.

\section{Materials and methods}

\section{Case Selection}

154 consecutive cases of endometrial carcinoma with available fresh frozen tissue and formalinfixed, paraffin-embedded tissue were retrieved from the tumor bank of the University of Texas M.D. Anderson Cancer Center Department of Pathology. The study was approved by the Institutional Review Board. The study cohort was composed of 100 pure endometrioid carcinomas (6 FIGO grade 1, 78 FIGO grade 2, and 16 FIGO grade 3) and 54 nonendometrioid carcinomas consisting of 13 carcinosarcomas, 10 undifferentiated carcinomas, 4 clearcell carcinomas, 1 serous carcinoma, and 26 mixed endometrioid/non-endometrioid carcinomas. Mixed carcinomas were designated as tumors with an endometrioid component and any amount of at least one other non-endometrioid histotype, including 
clear-cell carcinoma, serous carcinoma, undifferentiated carcinoma, carcinoma with sarcomatoid features, or carcinoma with neuroendocrine features. In these cases, discrete areas of endometrioid and nonendometrioid carcinoma could not be well demarcated. Rather, the majority of tumor exhibited overlapping features of two or more conventional histotypes. An additional cohort of 33 non-endometrioid carcinomas was identified subsequently from the files of the Department of Pathology, consisting of 18 carcinosarcomas, 2 clear-cell carcinomas, 8 serous carcinomas, and 5 mixed endometrioid/nonendometrioid carcinomas. Only paraffin-embedded tissue was available for these 33 cases.

\section{Immunohistochemistry}

Immunohistochemistry was performed on $4 \mu \mathrm{m}$ sections of formalin-fixed, paraffin-embedded tumors. Deparaffinization and rehydration of the tissue sections were carried out, followed by antigen retrieval at $100{ }^{\circ} \mathrm{C}$ for $20 \mathrm{~min}$ with Tris-EDTA buffer, $\mathrm{pH}$ 6.0. Endogenous peroxidase was blocked with $3 \%$ peroxide for $5 \mathrm{~min}$. Primary antibodies included PTEN (Dako, clone 6H2.1; 1:100 dilution), MLH1 (BD Biosciences, clone G168-15; 1:25 dilution), MSH2 (Calbiochem, clone FE11; 1:100 dilution), PMS2 (BD Biosciences, clone A16-4; 1:125 dilution), and MSH6 (BD Biosciences, clone 44; 1:300 dilution). Primary antibody detection was performed using a polymer system (Bond Polymer Refine Detection, Leica). Staining development was achieved by incubation with DAB and DAB Enhancer. PTEN immunohistochemistry was scored by two different pathologists (BD and $\mathrm{RB}$ ) as positive, negative, and heterogeneous (both positive and negative foci within the same tumor section; Figure 1). This scoring system has been previously described and shown to be reproducible. ${ }^{28}$ MLH1, MSH2, MSH6, and PMS2 immunohistochemistry was scored as positive or negative. Complete absence of MMR protein expression was required in order for a case to be designated as MMR negative. Stromal cells served as an internal positive control.

\section{MLH1 Methylation}

For cases in which there was immunohistochemical loss of MLH1 protein expression, PCR-based MLH1 promoter methylation analysis was performed. DNA was isolated from formalin-fixed, paraffin-embedded tissue sections that were microdissected with a scalpel blade to provide relatively pure tumor samples for analysis. Isolated DNA was treated with bisulfite to convert unmethylated cytosine nucleotides to uracil using the Zymo EZ DNA MethylationGold Kit according to the manufacturer's instructions (Zymo Research, Orange, CA, USA). Methylation of MLH1 was assessed by methylation-specific PCR followed by capillary electrophoresis using FAM-labeled reverse primer and unlabelled forward primers (Integrated DNA Technology). The primer sequences were the following: methylated forward, 5'-GATAGCGATTTTTAACGC-3', unmethylated forward, $5^{\prime}$-AGAGTGGATAGTGATTTTTAATGT- $3^{\prime}$ and labeled reverse primer, 5'- FAM-TCTATAAATTAC TAAATCTCTTC- $3^{\prime}$. The forward primers were designed to distinguish the methylated amplicon from the unmethylated by difference in size. The
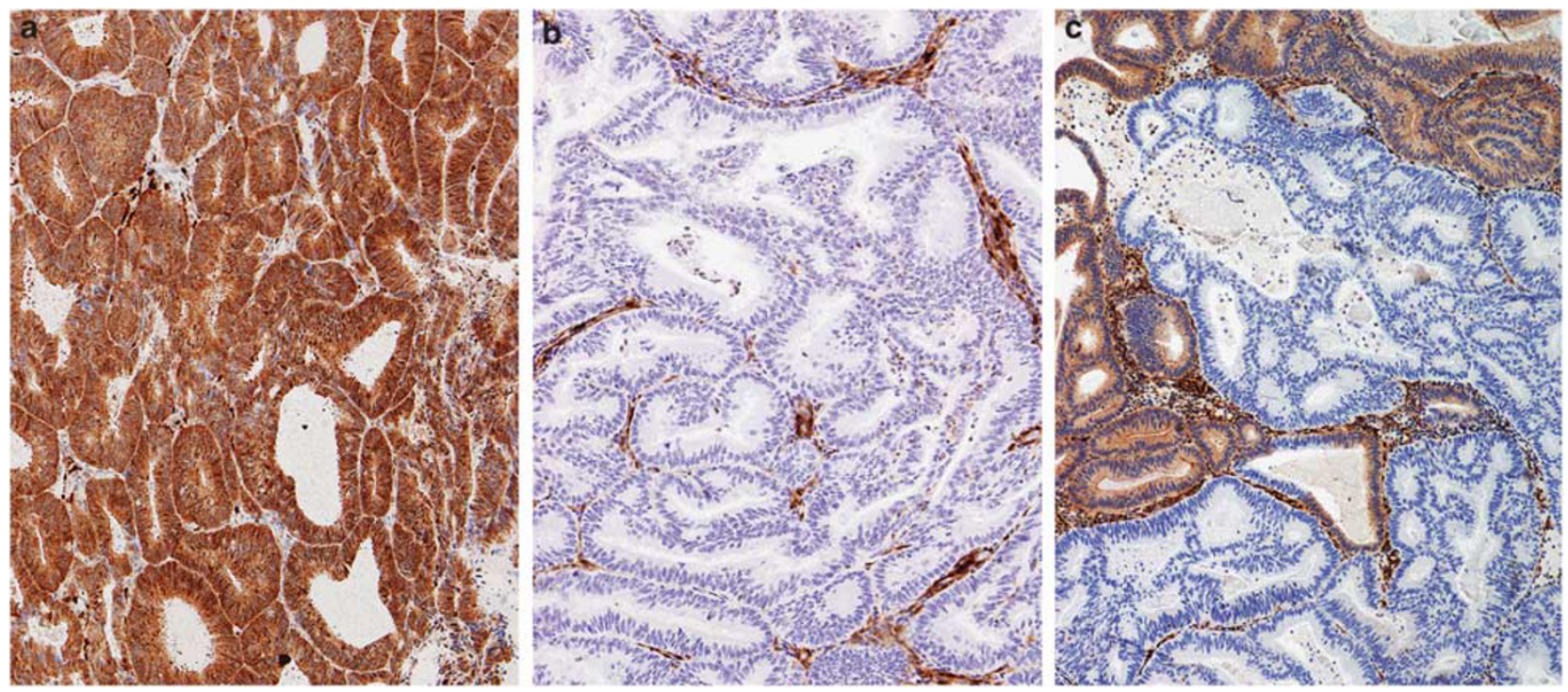

Figure 1 Three-tiered (positive, negative, heterogeneous) PTEN immunohistochemistry scoring. (a) Diffuse positive cytoplasmic PTEN staining is present in the majority $(>90 \%)$ of tumor cells. (b) For PTEN-negative tumors, no or only scattered tumor cells $(<1 \%)$ have cytoplasmic staining. Stromal cells serve as an internal positive control. (c) PTEN heterogeneous tumors have distinct positive and negative foci. All photomicrographs are of $\times 10$ magnification. 
bisulfite-treated DNA was then subjected to PCR using primers specific for methylated and unmethylated DNA. The methylated PCR product of $85 \mathrm{bp}$ was separated from unmethylated PCR product of $91 \mathrm{bp}$ by capillary electrophoresis using an ABI Prism 3130 Genetic Analyzer. Chromatograms for tumor were compared with those generated for the RKO colon carcinoma cell line (positive control known to have loss of MLH1 protein due to MLH1 promoter methylation) and the leukemia cell line K562 (negative control with no MLH1 methylation). Tumors with MLH1 immunohistochemical loss and presence of $M L H 1$ methylation were designated as likely sporadic, while tumors with MLH1 loss and absence of MLH1 methylation were designated as likely Lynch Syndrome.

\section{PTEN Sequencing}

Using standard methods, genomic DNA was isolated from fresh, frozen tumors. Bidirectional sequencing of PTEN exons 1-8 was carried out at the Human Genome Sequencing Center at Baylor College of Medicine using intron-based, exon-specific primers. PCR reactions were performed in $8 \mu \mathrm{l}$ containing $10 \mathrm{ng}$ of genomic DNA, $0.4 \mu \mathrm{M}$ oligonucleotide primers, and $0.7 \times$ Qiagen PCR HotStar Taq Master Mix containing buffer and polymerase. Cycling parameters were $95{ }^{\circ} \mathrm{C}-15 \mathrm{~min}$, then $95^{\circ} \mathrm{C}-45 \mathrm{~s}$, $60{ }^{\circ} \mathrm{C}-45 \mathrm{~s}$, and $72{ }^{\circ} \mathrm{C}-45 \mathrm{~s}$ for 40 cycles followed by a final extension at $72{ }^{\circ} \mathrm{C}$ for $7 \mathrm{~min}$, After thermocycling, $5 \mu$ l of a 1:15 dilution of Exo-SAP was added to each well, and reactions incubated at $37^{\circ} \mathrm{C}$ for $15 \mathrm{~min}$ before inactivation at $80^{\circ} \mathrm{C}$ for $15 \mathrm{~min}$. Reactions were diluted by $0.6 \times$ and $2 \mu \mathrm{l}$ were combined with $5 \mu \mathrm{l}$ of 1/64th Applied Biosystems (AB) BigDye sequencing reaction mix and cycled as above for 25 cycles. Reactions were precipitated with ethanol, re-suspended in $0.1 \mathrm{mM}$ EDTA, and loaded on $\mathrm{AB}$ 3730XL sequencing instruments using the Rapid36 run module and 3xx base-caller. SNP Detector software was used to identify SNPs. Identified mutations were verified by bidirectional re-sequencing of the original DNA sample. Sequencing of exon 9 for all tumors was performed at the University of Texas M.D. Anderson Cancer Center Molecular Diagnostics Laboratory. Two microliters of DNA were used to amplify exon 9 of PTEN using M13-tagged published primers. Reaction mixtures of $50 \mu \mathrm{l}$ contained $1 \mathrm{mM}$ dNTPs, $2.5 \mathrm{mM} \mathrm{MgCl} 2,0.2 \mathrm{uM}$ primers, $1.5 \mathrm{U}$ Ampli-Taq Gold 360 Polymerase (Applied Biosystems) were amplified with the following PCR conditions: an initial 10-min activation at $95{ }^{\circ} \mathrm{C}$ followed by 40 cycles of $30 \mathrm{~s}$ at $95^{\circ} \mathrm{C}$; $30 \mathrm{~s}$ at $50^{\circ} \mathrm{C} ; 30 \mathrm{~s}$ at $72^{\circ} \mathrm{C}$, and a final extension of $10 \mathrm{~min}$ at $72{ }^{\circ} \mathrm{C}$. PCR products were purified (Agencourt Ampure, Beckman Coulter) before being loaded on an ethidium bromide-stained agarose gel. Fluorescent-based automated cycle sequencing was performed by the dye-terminator method using a multi-capillary sequencer (ABI 3130 Genetic Analyzer; PE Applied Biosystems) according to the manufacturer's protocol (BigDye Terminator v1.1 Ready Reaction Cycle Sequencing Kit; PE Applied Biosystems). Briefly, reaction tubes (total volume $20 \mu \mathrm{l}$ ) containing $100 \mathrm{ng}$ of the purified PCR product, $3.2 \mathrm{pmol}$ of either the sense or antisense M13 primer and $6 \mu \mathrm{l}$ of the sequencing mixture were placed in a DNA thermal cycler and cycled for 25 cycles at $96{ }^{\circ} \mathrm{C}$ for $10 \mathrm{~s}, 58^{\circ} \mathrm{C}$ for $5 \mathrm{~s}, 60^{\circ} \mathrm{C}$ for $4 \mathrm{~min}$, and final hold at $4{ }^{\circ} \mathrm{C}$. Sequencing reactions were purified using the Qiagen DyeEx purification kit (Qiagen) as per the manufacturer's protocol. The resulting data were analyzed by Seqscape software (PE Applied Biosystems).

\section{Statistics}

Statistical analyses were performed using SPSS 17.0 (Chicago, IL, USA). Statistical comparisons were carried out using the Fisher's exact test. A $P$-value of $<0.05$ was considered significant.

\section{Results}

Table 1 shows some of the clinical and pathological features of patients in the study. The average age of patients with non-endometrioid tumors is significantly higher than the age of patients with pure endometrioid tumors $(P<0.0001)$. In addition, a significantly greater proportion of non-endometrioid tumors is of advanced stage (FIGO stage III or IV) compared with the pure endometrioid tumors $(P=0.0001)$. These characteristics are consistent with what is well known for endometrial cancer.

A summary of the MMR status for the endometrial carcinomas is presented according to tumor histotype and grade in Table 2. Loss of MMR protein was more frequent in the pure endometrioid group $(P=0.0001)$. This difference was predominantly

Table 1 Characteristics of endometrial cancer patients

\begin{tabular}{lccc}
\hline & $\begin{array}{c}\text { Pure endometrioid } \\
(\mathrm{n}=100)\end{array}$ & $\begin{array}{l}\text { Non-endometrioid } \\
(\mathrm{n}=87)\end{array}$ & $\begin{array}{c}\text { All cases } \\
(\mathrm{n}=187)\end{array}$ \\
\hline $\begin{array}{l}\text { Age (years) } \\
\text { Mean }\end{array}$ & 60.5 & & \\
Median & 61.1 & 67.9 & 64.0 \\
Range & $28.4-91.0$ & $28.1-92.4$ & 64.3 \\
& & & \\
a Stage & $65(65 \%)$ & $26(30 \%)$ & $91(49 \%)$ \\
1 & $10(10 \%)$ & $11(13 \%)$ & $21(11 \%)$ \\
2 & $19(19 \%)$ & $31(36 \%)$ & $50(27 \%)$ \\
3 & $6(6 \%)$ & $19(22 \%)$ & $25(13 \%)$ \\
4 & & & \\
\hline
\end{tabular}

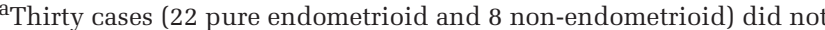
have grossly positive lymph nodes intra-operatively, and lymphadenectomy was thus not performed. These cases are staged according to the hysterectomy specimen as Stage I $(n=22)$, Stage II $(n=6)$, or Stage III $(n=2)$ 
Table 2 Overview of endometrial carcinomas with immunohistochemical loss of MLH1, MSH2, MSH6, or PMS2

\begin{tabular}{|c|c|c|c|c|c|}
\hline $\begin{array}{l}\text { Tumor histotype } \\
\text { and grade (n) }\end{array}$ & $\begin{array}{c}\text { Likely sporadic: } \\
\text { MLH1 loss with } \\
\text { MLH1 methylation, } \\
\text { n }(\%)\end{array}$ & $\begin{array}{c}\text { Likely Lynch } \\
\text { Syndrome: MLH1 } \\
\text { loss with no MLH1 } \\
\text { methylation, n (\%) }\end{array}$ & $\begin{array}{c}\text { Likely Lynch } \\
\text { Syndrome: MSH2, } \\
\text { MSH6, or PMS2 } \\
\text { loss, n (\%) }\end{array}$ & $\begin{array}{c}\text { Inconclusive } \\
\text { (MLH1 IHC loss but } \\
\text { methylation } \\
\text { analysis did not } \\
\text { work), } \mathrm{n}(\%)\end{array}$ & $\begin{array}{l}\text { Total cases with } \\
\text { loss of MMR } \\
\text { proteins, } \mathrm{n}(\%)\end{array}$ \\
\hline All cases (187) & $25(13 \%)$ & $7(4 \%)$ & $11(6 \%)$ & $3(2 \%)$ & $46(25 \%)$ \\
\hline Pure endometrioid (100) & $20(20 \%)$ & $6(6 \%)$ & $8(8 \%)$ & $2(2 \%)$ & $36(36 \%)$ \\
\hline Grade $1(6)$ & $0(0 \%)$ & $0(0 \%)$ & $0(0 \%)$ & & $0(0 \%)$ \\
\hline Grade 2 (78) & $16(16 \%)$ & $4(4 \%)-$ MLH1 $\times 4$ & $\begin{array}{c}7(7 \%)-\mathrm{MSH} 2 \times 1 \\
\text { MSH6 } \times 2 \\
\text { PMS } 2 \times 4\end{array}$ & $1(1 \%)$ & $28(28 \%)$ \\
\hline Grade 3 (16) & $4(4 \%)$ & $2(2 \%)-\mathrm{MLH} 1 \times 2$ & $1(1 \%)-P M S 2 \times 1$ & $1(1 \%)$ & $8(8 \%)$ \\
\hline Non-endometrioid (87) & $5(6 \%)$ & $1(1 \%)$ & $3(3 \%)$ & $1(1 \%)$ & $10(11 \%)$ \\
\hline Mixed (31) & $2(2 \%)$ & $1(1 \%)-$ MLH $1 \times 1$ & $3(3 \%)-\mathrm{MSH} 6 \times 3$ & $1(1 \%)^{a}$ & $7(8 \%)$ \\
\hline Undifferentiated (10) & $1(1 \%)$ & $0(0 \%)$ & $0(0 \%)$ & & $1(1 \%)$ \\
\hline Carcinosarcoma (31) & $2(2 \%)$ & $0(0 \%)$ & $0(0 \%)$ & & $2(2 \%)$ \\
\hline Clear cell (6) & $0(0 \%)$ & $0(0 \%)$ & $0(0 \%)$ & & $0(0 \%)$ \\
\hline Serous (9) & $0(0 \%)$ & $0(0 \%)$ & $0(0 \%)$ & & $0(0 \%)$ \\
\hline $\begin{array}{l}P \text {-value (endometrioid vs } \\
\text { non-endometrioid) }\end{array}$ & 0.0047 & 0.1234 & 0.2253 & & 0.0001 \\
\hline
\end{tabular}

Data summarized are for $n=187$ endometrial carcinomas with paraffin-embedded tissue available for immunohistochemistry.

${ }^{\mathrm{a}}$ The non-endometrioid carcinoma with MLH1 IHC loss but inconclusive methylation analysis was a carcinosarcoma.

Table 3 Correlation between PTEN status (by immunohistochemistry and by mutation) with MMR immunohistochemistry status ${ }^{\mathrm{a}}$

\begin{tabular}{|c|c|c|c|c|c|c|}
\hline & $\begin{array}{l}\text { Pure endometrioid } \\
\qquad(\mathrm{n}=100)\end{array}$ & P-value & $\begin{array}{l}\text { Non-endometrioid } \\
\quad(\mathrm{n}=54)\end{array}$ & $\mathrm{P}$-value & $\begin{array}{l}\text { All tumors } \\
(\mathrm{n}=154)\end{array}$ & $\mathrm{P}$-value \\
\hline $\begin{array}{l}\text { Cases with PTEN loss by IHC as a } \\
\text { subset of cases with intact MMR }\end{array}$ & $45 / 64(70 \%)$ & 0.2285 & $15 / 45(33 \%)$ & 0.0030 & $60 / 109(56 \%)$ & 0.0005 \\
\hline $\begin{array}{l}\text { Cases with PTEN loss by IHC as a } \\
\text { subset of cases with MMR loss }\end{array}$ & $30 / 36(83 \%)$ & & 8/9 (89\%) & & $38 / 45(84 \%)$ & \\
\hline $\begin{array}{l}\text { Cases with PTEN mutation as a } \\
\text { subset of cases with intact MMR }\end{array}$ & $30 / 64(47 \%)$ & 0.3029 & $8 / 45(18 \%)$ & 0.0009 & $38 / 109$ (35\%) & 0.0023 \\
\hline $\begin{array}{l}\text { Cases with PTEN mutation as a } \\
\text { subset of cases with MMR loss }\end{array}$ & $21 / 36(58 \%)$ & & 7/9 (78\%) & & $28 / 45(62 \%)$ & \\
\hline
\end{tabular}

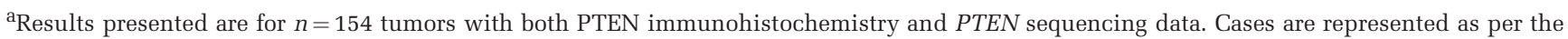
following example: Of 100 pure endometrioid cases examined, 64 have intact MMR expression. Of these, 45 (70\%) have PTEN loss by immunohistochemistry. The $P$-values are derived from a Fisher's exact test comparison of proportion cases with PTEN loss by immunohistochemistry (or PTEN mutation) between groups with intact MMR and MMR loss.

attributable to a greater proportion of pure endometrioid tumors with MLH1 methylation compared with non-endometrioid tumors. Notably, among the non-endometrioid group, mixed carcinomas were the histotype that most commonly had loss of a MMR protein.

Table 3 shows the correlation between PTEN loss detected by immunohistochemistry (cases scored as negative or heterogeneous) vs PTEN mutation in tumors with intact MMR and loss of a MMR protein. In pure endometrioid tumors, the frequency of PTEN loss measured by immunohistochemistry $(P=0.2285)$ and mutation $(P=0.3029)$ did not differ significantly between the MMR intact or MMR loss groups, suggesting that PTEN loss, no matter the mechanism, is independent of MMR status in endometrioid-type endometrial carcinoma. However, in non-endometrioid tumors, both PTEN immunohistochemical loss $(P=0.0030)$ and PTEN loss due to mutation $(P=0.0009)$ were significantly more frequent in tumors with loss of MMR. Interestingly, seven of eight of these non-endometrioid tumors with PTEN protein loss by immunohistochemistry were tumors with PTEN mutations.

Table 4 summarizes the specific nature of PTEN mutations in endometrial carcinomas with MLH1 protein loss due to MLH1 methylation (sporadic) and in endometrial carcinomas with immunohistochemical loss of MLH1 (MLH1 unmethylated), MSH2, MSH6, and PMS2 (likely Lynch Syndrome associated). It is interesting to note the difference in the distribution of PTEN gene insertions/deletions in pure endometrioid tumors with PTEN loss and loss of MMR, which concentrate in exons 7 and 8 compared with pure endometrioid tumors with PTEN loss and intact MMR, which seem to span over the entire PTEN gene. A similar trend is observed in the non-endometrioid tumors. In the non-endometrioid group, tumors with exon 7 , exon 8 and exon 8 boundary PTEN sequence abnormalities 
Table 4 Nature of PTEN mutations according to MMR status

Pure Endometrioid

\begin{tabular}{|c|c|c|c|c|}
\hline $\begin{array}{l}\text { PTEN mutation and } \\
\text { immunohistochemistry status }\end{array}$ & MMR status & $\begin{array}{c}\text { Total number of } \\
\text { sequence } \\
\text { abnormalitites }\end{array}$ & $\begin{array}{l}\text { Number of } \\
\text { single base } \\
\text { substitutions }\end{array}$ & Number of insertions/deletions \\
\hline \multirow{3}{*}{$\begin{array}{l}\text { PTEN mutation present and } \\
\text { PTEN loss by } \\
\text { immunohistochemistry }\end{array}$} & $\begin{array}{l}\text { MLH1 loss, } \\
\text { MLH1 methylated }\end{array}$ & 17 & 11 & 6: exon 7 (2) and exon 8 (4) \\
\hline & $\begin{array}{l}\text { MMR loss including } \\
\text { MLH1 unmethylated }\end{array}$ & 8 & 3 & 5: exon 6 (1) exon 7 (3) and exon 8 (1) \\
\hline & MMR intact & 35 & 20 & $\begin{array}{l}\text { 15: exon } 1 \text { (4), exon } 3 \text { boundary (1), exon } 5(4) \text {, } \\
\text { exon } 6(2) \text {, exon } 7(2) \text {, exon } 8(2)\end{array}$ \\
\hline \multirow{3}{*}{$\begin{array}{l}\text { PTEN mutation present and } \\
\text { no PTEN loss by } \\
\text { immunohistochemistry }\end{array}$} & $\begin{array}{l}\text { MLH1 loss, } \\
\text { MLH1 methylated }\end{array}$ & 2 & 1 & 1: exon 5 \\
\hline & $\begin{array}{l}\text { MMR loss including } \\
\text { MLH1 unmethylated }\end{array}$ & 1 & 0 & 1: exon 7 \\
\hline & MMR intact & 3 & 2 & 1: exon 1 \\
\hline
\end{tabular}

Non-endometrioid

\begin{tabular}{llccc}
\hline $\begin{array}{l}\text { PTEN mutation and } \\
\text { immunohistochemistry status }\end{array}$ & MMR status & $\begin{array}{c}\text { Total number of } \\
\text { sequence } \\
\text { abnormalitites }\end{array}$ & $\begin{array}{c}\text { Number of } \\
\text { single base } \\
\text { substitutions }\end{array}$ & Number of insertions/deletions \\
\hline $\begin{array}{l}\text { PTEN mutation present and } \\
\text { PTEN loss by } \\
\text { immunohistochemistry }\end{array}$ & $\begin{array}{l}\text { MLH1 loss, } \\
\text { MLH1 methylated }\end{array}$ & 3 & 1 & 2: exon 8 and exon 9 \\
& $\begin{array}{l}\text { MMR loss including } \\
\text { MLH1 unmethylated }\end{array}$ & 5 & 2 & 3: exon 5, exon 7, exon 8 boundary \\
& $\begin{array}{l}\text { MMR intact } \\
\text { MLH1 loss, } \\
\text { MLH1 methylated }\end{array}$ & 7 & 3 & 4: exon 1 (1), exon 4 (1), exon 8 (2) \\
$\begin{array}{l}\text { PTEN mutation present and } \\
\text { no PTEN loss by } \\
\text { immunohistochemistry }\end{array}$ & 0 & 0 & 0 \\
& $\begin{array}{l}\text { MMR loss including } \\
\text { MLH1 unmethylated }\end{array}$ & 0 & 0 & 0 \\
\hline
\end{tabular}

Results are summarized from $n=154$ endometrial carcinomas with frozen tissue available for PTEN sequencing.

correspond to two mixed endometrioid/nonendometrioid carcinomas and one undifferentiated carcinoma. Of the 11 PTEN insertions/deletions that occurred in pure endometrioid tumors with loss of MMR (sporadic tumor or Lynch Syndrome), 8 were in poly A5 and A6 tracts. Of the five PTEN insertions/deletions that occurred in non-endometrioid tumors with loss of MMR, two were in poly A5 and A6 tracts.

For Table 5, PTEN immunohistochemistry and gene mutations are charted according to the specific MMR groups, MLH1 loss due to MLH1 methylation (likely sporadic endometrial carcinoma) and loss of MLH1 (MLH1 unmethylated), MSH2, MSH6, or PMS2 (likely Lynch Syndrome-associated endometrial carcinoma). In both pure endometrioid and non-endometrioid tumors with MMR loss and PTEN loss due to mutation, MLH1 loss secondary to MLH1 methylation occurs with similar frequency as MMR loss likely due to Lynch Syndrome $28 \%$ vs $22 \%$ in endometrioid and 33\% vs 33\% in non-endometrioid tumors). Thus, assessment of PTEN, either by immunohistochemistry or sequencing, is not useful in distinguishing sporadic endometrial carcinomas with MLH1 methylation from Lynch Syndromeassociated endometrial carcinomas with loss of MLH1, MSH2, MSH6, or PMS2.

We next investigated whether PTEN status could specifically distinguish between sporadic endometrial carcinomas with MLH1 methylation $(n=24)$ and MLH1 protein loss by immunohistochemistry and likely Lynch Syndrome-associated endometrial carcinomas with MLH1 loss and no MLH1 methylation $(n=7)$. PTEN mutation was detected in $14 / 24$ of the sporadic cases and $5 / 7$ of the likely Lynch Syndrome cases, while PTEN 
Table 5 PTEN status in pure endometrioid and non-endometrioid endometrial carcinomas with immunohistochemical loss of a MMR protein

\begin{tabular}{|c|c|c|c|}
\hline Total $(\mathrm{n}=154)$ & $\begin{array}{l}\text { Likely sporadic: } \\
\text { MLH1 loss, } M L H 1 \\
\text { methylated, n (\%) }\end{array}$ & $\begin{array}{c}\text { Likely Lynch Syndrome: } \\
\text { MMR loss, MLH1 } \\
\text { unmethylated, n (\%) }\end{array}$ & $\begin{array}{l}\text { Total cases with loss } \\
\text { of MMR protein, } \mathrm{n}(\%)\end{array}$ \\
\hline \multicolumn{4}{|l|}{ Pure endometrioid $(\mathrm{n}=100)$} \\
\hline PTEN mutation and PTEN loss & $10(28 \%)$ & $8(22 \%)$ & $18(50 \%)$ \\
\hline PTEN wt and PTEN loss & $7(19 \%)$ & $3(8 \%)$ & $10(28 \%)$ \\
\hline PTEN mutation and no PTEN loss & $1(3 \%)$ & $1(3 \%)$ & $2(6 \%)$ \\
\hline PTEN wt and no PTEN loss & $2(6 \%)$ & $2(6 \%)$ & $4(11 \%)$ \\
\hline \multirow[t]{2}{*}{ Methylation analysis inconclusive } & & & $2(6 \%)$ \\
\hline & & & $36(100 \%)$ \\
\hline \multicolumn{4}{|l|}{ Non-endometrioid $(\mathrm{n}=54)$} \\
\hline PTEN mutation and PTEN loss & $3(33 \%)$ & $3(33 \%)$ & $6(67 \%)$ \\
\hline PTEN wt and PTEN loss & $0(0 \%)$ & $1(11 \%)$ & $1(11 \%)$ \\
\hline PTEN mutation and no PTEN loss & $0(0 \%)$ & $0(0 \%)$ & $0(0 \%)$ \\
\hline PTEN wt and no PTEN loss & $1(11 \%)$ & $0(0 \%)$ & $1(11 \%)$ \\
\hline \multirow[t]{2}{*}{ Methylation analysis inconclusive } & & & $1(11 \%)$ \\
\hline & & & $9(100 \%)$ \\
\hline
\end{tabular}

Results presented are for $n=154$ tumors with both PTEN immunohistochemistry and PTEN sequencing data.

immunohistochemical loss was present in 20/24 sporadic tumors and 5/7 likely Lynch Syndrome tumors (data not shown). There was no statistical difference between the sporadic and Lynch Syndrome groups for either PTEN mutation or PTEN immunohistochemical loss. The numbers are small, but we can preliminarily conclude that PTEN assessment cannot reliably distinguish between these two groups of endometrial carcinomas with MLH1 loss by immunohistochemistry.

Figure 2 summarizes how PTEN immunohistochemistry could possibly be used in the clinical setting as a predictor of MMR status. For patients with pure endometrioid endometrial carcinoma, PTEN is not a good predictor of MMR status $(P=0.2285, \quad$ specificity $=30 \%, \quad$ sensitivity $=83 \%$, $\mathrm{PPV}=75 \%, \mathrm{NPV}=40 \%$ ). For patients with nonendometrioid carcinomas, however, positive PTEN immunohistochemistry is associated with a high likelihood of intact expression of MLH1, MSH2, MSH6, and PMS2 $(P=0.0043$, specificity $=69 \%$, sensitivity $=80 \%, \mathrm{PPV}=96 \%, \mathrm{NPV}=2 \%$ ). The two PTEN-positive, non-endometrioid carcinomas with loss of MMR were carcinosarcomas with immunohistochemical loss of MLH1 and the presence of MLH1 methylation. Thus, none of the PTEN-positive, non-endometrioid carcinomas were associated with Lynch Syndrome. This figure also highlights that PTEN status is associated with MMR, but only in a narrow setting; specifically, non-endometrioid endometrial carcinomas with positive PTEN expression nearly always have retained expression of MMR proteins. Note that PTEN gene sequencing is omitted from this clinical schematic. From Tables 3 and 4, PTEN mutations and PTEN immunohistochemistry loss both had similar profiles in pure endometrioid and non-endometrioid tumors with respect to MMR status. As we have previously shown, ${ }^{8}$ PTEN immunohistochemistry outperforms PTEN sequencing for detecting PTEN loss in endometrial carcinoma. This, plus the fact that PTEN immunohistochemistry is less expensive than sequencing and more widely available in pathology laboratories, led us to include only PTEN immunohistochemistry in the clinical schematic for Figure 2. In addition to PTEN immunohistochemistry, we examined other clini$\mathrm{cal} /$ pathological factors, such as patient age and tumor histology, which may be important in distinguishing Lynch Syndrome-associated endometrial carcinoma from sporadic tumors. Eighteen patients had loss of expression of a mismatch repair protein that is indicative of likely Lynch Syndrome $(n=14$ patients with pure endometrioid tumors; $n=4$ patients with non-endometrioid tumors). For patients with pure endometrioid tumors, the mean/ median age was 64.1/64.5 years, and for patients with non-endometrioid tumors, the mean/median age was 64.3/68.0 years. These probable Lynch Syndrome patients are not significantly younger than those for the entire patient population (Table 1). This is not surprising, given that a recent population-based study of women with endometrial cancer found that the median age of the Lynch Syndrome patients (59 years) was nearly equivalent to that of the patients with sporadic endometrial cancer (61 years). ${ }^{1}$

Some authors have suggested that microscopic morphological features, including 'morphological ambiguity', ${ }^{29,30}$ may be a clue to identifying Lynch Syndrome-associated endometrial carcinomas. Among the eight non-endometrioid carcinomas with PTEN loss and MMR loss (Figure 2), four are likely Lynch Syndrome associated (Table 6). Each of these tumors has mixed endometrioid/non-endometrioid histology ((1) mixed carcinoma with endometrioid, serous and undifferentiated components; (2) mixed carcinoma that is predominantly high-grade endometrioid with a minor serous component; (3) mixed carcinoma with high-grade endometrioid and 


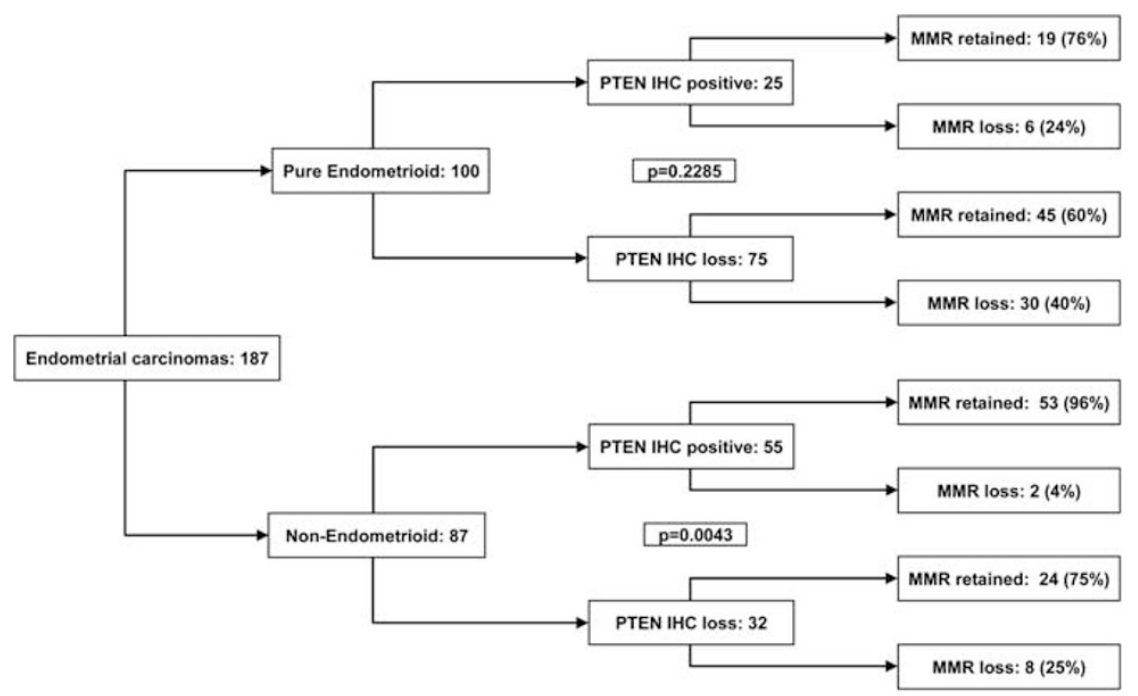

Figure 2 PTEN immunohistochemistry as a predictor of MMR status for endometrial carcinoma. The $P$-values are derived from a Fisher's exact test comparison of proportion cases with retained MMR expression between groups with positive PTEN immunohistochemistry and with loss of PTEN by immunohistochemistry. For endometrioid carcinomas, PTEN immunohistochemical status cannot reliably distinguish between tumors with intact MMR from tumors with loss of MMR. However, for non-endometrioid carcinomas, positive PTEN immunohistochemistry is highly correlated to retained positive expression of MMR proteins. Thus, for non-endometrioid endometrial carcinomas, PTEN immunohistochemistry is a single biomarker that can potentially be used to help identify which patients should receive further workup for Lynch Syndrome. In this schematic, 'PTEN IHC loss' represents cases where PTEN immunohistochemistry was scored as either negative or heterogeneous.

clear-cell carcinoma components; and (4) mixed low-grade endometrioid carcinoma and sarcomatoid carcinoma). From Table 6, it is evident that the majority of endometrial carcinomas with mismatch repair defects have endometrioid histology. Six out of eight non-endometrioid carcinomas with mismatch repair defects have an endometrioid component microscopically. In our data set, mixed carcinomas with endometrioid components of likely Lynch Syndrome patients represent only 4/31 (13\%) of all mixed carcinomas with endometrioid components and only 4/18 (22\%) of all likely Lynch Syndrome patients (the remaining 14 probable Lynch Syndrome patients have pure endometrioid tumors). Therefore, 'morphological ambiguity' of endometrial tumors does not appear to be a sufficiently specific or sensitive feature for identifying patients with possible Lynch Syndrome.

\section{Discussion}

Our study is the first to compare PTEN loss by mutation vs PTEN protein loss by immunohistochemistry in a set of endometrial carcinomas that are well characterized for defects in DNA mismatch repair. Among pure endometrioid tumors, neither PTEN mutation nor PTEN protein loss showed a correlation with MMR loss (Table 3). As previously noted, some investigators found a correlation between MSI-high and PTEN mutations ${ }^{15,16,31}$ or MSIhigh and PTEN protein loss, ${ }^{18,20}$ while others did not. ${ }^{13,14}$ These discrepancies between different studies may be due to the fact that some PTEN mutations are predisposed to arise in the setting of MSI-high, while others may arise independently of defects in DNA mismatch repair. Although insertions/deletions in pure endometrioid tumors with PTEN mutations and MMR loss showed a predilection for poly A tracts within exons 7 and 8 (Table 4), a trend also observed by others, ${ }^{15,16,31}$ single base substitutions (14/25) occurred with comparable frequency to insertions/deletions (11/ 25 ) in this group of tumors. This finding again supports the notion that not all PTEN mutations arise as a consequence of MSI-high.

In non-endometrioid tumors, both PTEN protein loss and PTEN mutations occurred more frequently in tumors with loss of MMR, with PTEN loss being attributable to mutations in the majority of cases (Table 3). Similar to the pure endometrioid tumors, some PTEN insertions/deletions occured in exons 7 and 8 in cases with MMR PTEN loss and also appeared with comparable frequency as single base substitutions (Table 4). Specific type of MMR loss, regardless of MLH1 methylation status, did not have an impact on PTEN mutations or PTEN protein loss in pure endometrioid nor non-endometrioid tumors (Table 5). Although this is a novel finding, it should be cautioned that, as more endometrial cancer patients are tested for defects in MMR and more data accumulate, specific types of PTEN mutation may indeed be associated with specific defects in MMR that lead to MSI-high.

Our results demonstrate that MMR loss occurs more frequently in pure endometrioid carcinomas compared with non-endometrioid carcinomas (Table 2), which has previously been established 
Table 6 Overview of endometrial carcinomas by tumor histotype with immunohistochemical loss of PTEN and of MLH1, MSH2, MSH6, or PMS2 ${ }^{\mathrm{a}}$

\begin{tabular}{|c|c|c|c|c|c|}
\hline PTEN IHC loss (n) & $\begin{array}{c}\text { Likely sporadic: } \\
\text { MLH1 loss with } \\
\text { MLH1 methylation, } \\
\text { n (\%) }\end{array}$ & $\begin{array}{c}\text { Likely Lynch } \\
\text { Syndrome: MLH1 } \\
\text { loss with no MLH1 } \\
\text { methylation, n (\%) }\end{array}$ & $\begin{array}{c}\text { Likely Lynch } \\
\text { Syndrome: } M S H 2 \text {, } \\
\text { MSH6, or PMS2 } \\
\text { loss, n (\%) }\end{array}$ & $\begin{array}{c}\text { Inconclusive } \\
\text { (MLH1 IHC loss but } \\
\text { methylation } \\
\text { analysis did not } \\
\text { work) n (\%) }\end{array}$ & $\begin{array}{c}\text { Total cases with } \\
\text { loss of MMR } \\
\text { proteins, } \mathrm{n}(\%)\end{array}$ \\
\hline All cases (107) & $20(19 \%)$ & $5(5 \%)$ & $10(9 \%)$ & $3(3 \%)$ & $38(35 \%)$ \\
\hline Pure endometrioid (75) & $17(23 \%)$ & $4(5 \%)$ & $7(9 \%)$ & $2(3 \%)$ & $30(40 \%)$ \\
\hline Non-endometrioid (32) & $3(9$ \%) & $1(3 \%)$ & $3(9 \%)$ & $1(3 \%)$ & $8(25 \%)$ \\
\hline Mixed with endometrioid (13) & $2(15 \%)$ & $1(8 \%)$ & $3(23 \%)$ & & $6(46 \%)$ \\
\hline Pure non-endometrioid (19) & $1(5 \%)$ & $0(0 \%)$ & $0(0 \%)$ & $1(5 \%)^{b}$ & $2(5 \%)$ \\
\hline
\end{tabular}

${ }^{a}$ Data summarized are for $n=187$ endometrial carcinomas with paraffin-embedded tissue available for immunohistochemistry. These include $n=100$ pure endometrioid and $n=87$ non-endometrioid carcinomas. The latter group contains $n=56$ pure non-endometrioid ( $n=9$ serous carcinomas, $n=6$ clear-cell carcinomas, $n=31$ carcinosarcomas, and $n=10$ undifferentiated carcinomas) and $n=31$ mixed carcinomas with combined endometrioid and non-endometrioid components.

${ }^{\mathrm{b}}$ The non-endometrioid carcinoma with MLH1 IHC loss but inconclusive methylation analysis was a carcinosarcoma.

by other investigators. ${ }^{20,24,26}$ In this study, this difference is primarily due to a high proportion of pure endometrioid tumors with MLH1 methylation, which is a reflection of the unselected cohort of endometrial carcinoma patients in which sporadic endometrial tumors (sporadic with MLH1 methylation and sporadic with intact MMR expression) are expected to predominate over Lynch Syndromeassociated tumors. Within the non-endometrioid group, mixed endometrioid/non-endometrioid carcinomas comprised the largest group with MMR defects (4/31 cases, $13 \%$ ), by far the most defects in MMR compared with the other non-endometrioid tumor types. In addition, these mixed tumors were the only subset within the non-endometrioid group to exhibit MMR protein loss without MLH1 methylation. Therefore, with regards to MMR, the mixed tumors are more like pure endometrioid tumors, as loss of MMR is more common in pure endometrioid than in nonendometrioid carcinomas. However, loss of MMR is still much more common in pure endometrioid tumors (34\% with loss of MLH1, MSH2, MSH6, or PMS2) than in mixed tumors. Similarly, we have previously shown that the mixed carcinomas as a group do exhibit PTEN sequence abnormalities and PTEN protein loss, but less often than in the pure endometrioid group. ${ }^{32}$ Some investigators have also shown that these mixed endometrioid/non-endometrioid carcinomas have a frequency of PIK3CA, PIK3R1, and PIK3R2 mutations more comparable with that of pure endometrioid tumors, ${ }^{33}$ while in other studies the frequency of PIK3CA mutations, particularly in exon 20, as well as p53 mutations, more closely paralleled that in pure non-endometrioid tumors. ${ }^{34}$ In aggregate, these results suggest that the mixed endometrioid/nonendometrioid carcinomas are a somewhat unique subset of endometrial carcinoma, sharing some molecular features not only with pure endometrioid carcinomas but also with pure non-endometrioid carcinomas.

Other non-endometrioid tumor types, particularly undifferentiated endometrial carcinomas and carcinosarcomas, also had loss of MMR, although it was uncommon. In these cases, the MMR defect was secondary to MLH1 methylation. Undifferentiated endometrial carcinomas have previously been found to be associated with MSI-high due to both sporadic MLH1 methylation ${ }^{4}$ and Lynch Syndrome. ${ }^{35}$ Given that the study cohort involves patients unselected for young age or positive family history, it is reasonable to conclude that the undifferentiated carcinomas in this study are not likely to be Lynch Syndrome associated.

It is worthwhile to note at this junction that many of the associations described above would be lost if endometrial carcinoma was considered as a single disease. This is a common problem in the scientific literature, and only a handful pathologists to date have attempted to use immunohistochemistry ${ }^{36}$ or gene expression profiling ${ }^{37,38}$ to classify endometrial cancers into categories based on prognostic outcome or gene abnormalities in key molecular pathways. This is particularly relevant from the point of view of cooperative group clinical trials, which typically do not take into account considerations of specific endometrial carcinoma histotypes as eligibility criteria. An example of the importance of this concept can certainly be seen in breast medical oncology, in which patients with estrogen receptorpositive breast carcinomas are treated very differently than those with HER2/neu-positive tumors or triple-negative tumors. These differences in treatment are supported by well-established and well-accepted genomic studies documenting the molecular complexity and heterogeneity of breast cancer. ${ }^{39}$ Similarly, in the gynecologic oncology community, there is much emerging interest in identifying ovarian/fallopian tube/primary peritoneal high-grade serous carcinomas with defects in homologous recombination DNA repair, as these tumors may be preferentially sensitive to poly (ADP-ribose) polymerase inhibitors. ${ }^{40}$

In order to identify endometrial carcinoma patients who should undergo genetic testing for MMR gene mutations (Lynch Syndrome), clinical screening guidelines, based on patient's age of $<50$ years and 
a personal and/or a family history of Lynch Syndromeassociated cancers, have been developed. ${ }^{41,42}$ Similarly, tumor topography, such as lower uterine segment location ${ }^{43}$ or histological features, such as the presence of tumor infiltrating lymphocytes and tumor heterogeneity, ${ }^{44}$ have been found to have an association with Lynch Syndrome. However, patient and/ or tumor characteristics are unable to identify Lynch Syndrome tumors with sufficient sensitivity and specificity. Furthermore, some endometrial tumors with MSH6 mutations may not be MSI-high and occur in patients $>50$ years with no significant family history. ${ }^{2,45}$ It has been shown that clinical screening tools that rely on young age of cancer diagnosis and family history, such as PREMM, MMRpredict, and MMRpro, perform poorly in predicting germline Lynch Syndrome mutations in women with endometrial cancer, ${ }^{46}$ suggesting that populationbased screening of these tumors should be implemented, with tumor tissue testing, rather than mutation testing, being the most cost-effective first step. ${ }^{47,48}$ Currently, although the majority of tumors with MMR genetic loss can be detected by immunohistochemistry for MLH1, MSH2, MSH6, and PMS2, a small percentage of tumors exhibit retained expression of non-functional MMR proteins. Therefore, concurrent testing with PCR-based MSI analysis is recommended. ${ }^{49,50}$ Tissue testing even with just immunohistochemistry incurs considerable costs, ${ }^{51}$ and implementation of national screening strategies may involve substantial logistical challenges. ${ }^{52,53}$ Furthermore, not all clinical pathology laboratories have the resources and/or the expertise to offer PCR-based testing.

In the unselected cohort of patients in our study, $14 \%$ of patients with pure endometrioid tumors and $5 \%$ of patients with non-endometrioid tumors were found to likely have Lynch Syndrome based on tissue testing studies (Table 2). All of the patients in the latter group had carcinomas with mixed endometrioid and non-endometrioid components. In terms of screening for Lynch Syndrome, if the goal of tumor screening is to capture all Lynch syndrome patients, then all tumors, no matter the histology, should be tested, as pure non-endometrioid tumors have been previously associated with Lynch Syndrome. ${ }^{4}$ However, if screening resources are more limited, acknowledging that the screening algorithm may miss a small number of women with Lynch Syndrome, our data suggest that patients with non-endometrioid carcinomas, and particularly those lacking any endometrioid components microscopically, may be excluded from testing. Alternatively, if all endometrial carcinoma patients are to be screened, PTEN immunohistochemistry (in combination with tumor histotype) may be used to refine the screening algorithm, by triaging endometrial tumors that require tissue testing. For non-endometrioid tumors with positive PTEN expression, 96\% also had retained MMR protein expression (Figure 2). Thus, a single immunohisto- chemistry test may be able to replace the use of four MMR immunohistochemical markers in the screening of $63 \%(55 / 87)$ of patients with non-endometrioid type endometrial carcinoma. It is important to note here that this study only accounted for endometrial tumor histology; important factors like family history of Lynch Syndrome-associated cancers and age may also be considered when contemplating tumor tissue testing of an individual patient to screen for Lynch Syndrome.

Note, however, that PTEN immunohistochemistry is not useful in distinguishing endometrial carcinomas with Lynch Syndrome-associated loss of MMR from sporadic tumors with MLH1 loss secondary to MLH1 methylation. For colorectal cancer, the presence of $B R A F$ mutation is indicative of a sporadic tumor, as $B R A F$ mutation can be present in tumors with $M L H 1$ methylation, ${ }^{54-56}$ but has not been reported in Lynch Syndrome-associated tumors. ${ }^{57}$ Assaying for $B R A F$ mutation is relatively simple, as mutations can be assessed in one hotspot. BRAF mutations are exceedingly rare in sporadic endometrial carcinoma, so this analysis does not aid in distinguishing sporadic from Lynch Syndrome-associated tumors. ${ }^{58,59}$ When The Cancer Genome Atlas has completed its genomic analyses for endometrial cancer, it is possible that mutation in an alternative gene or set of genes will be identified to be associated exclusively with sporadic or hereditary endometrial cancer.

In aggregate, the data summarized in this paper highlight the importance of accurate pathological classification of endometrial carcinoma into endometrioid and non-endometrioid types. Specifically, we found that retained PTEN expression by immunohistochemistry is very tightly linked to retained positive expression of the DNA MMR proteins MLH1, MSH2, MSH6, and PMS2 in non-endometrioid carcinomas, whereas PTEN expression is not associated with expression of MMR proteins in the endometrioid carcinomas. Therefore, PTEN immunohistochemistry, in combination with tumor histotype, may be a useful adjunct to clinical testing of endometrial carcinomas for Lynch Syndrome.

\section{Acknowledgements}

This work was supported by NIH 2P50 CA098258-06 SPORE in Uterine Cancer (to RRB) and Stand Up to Cancer/American Association for Cancer Research Dream Team Translational Cancer Research Grant, Grant No. SU2C-AACR- DT0209 (to RRB), and M.D. Anderson Cancer Center Support Grant (to CCSG) CA016672 from the National Institutes of Health.

\section{Disclosure/conflict of interest}

The authors declare no conflict of interest. 


\section{References}

1 Leenen $\mathrm{CH}$, van Lier MG, van Doorn $\mathrm{HC}$, et al. Prospective evaluation of molecular screening for Lynch syndrome in patients with endometrial cancer $</=70$ years. Gynecol Oncol 2012;125:414-420.

2 Hampel H, Frankel W, Panescu J, et al. Screening for Lynch syndrome (hereditary nonpolyposis colorectal cancer) among endometrial cancer patients. Cancer Res 2006;66:7810-7817.

3 Salvesen HB, MacDonald N, Ryan A, et al. Methylation of hMLH1 in a population-based series of endometrial carcinomas. Clin Cancer Res 2000;6:3607-3613.

4 Broaddus RR, Lynch HT, Chen LM, et al. Pathologic features of endometrial carcinoma associated with HNPCC: a comparison with sporadic endometrial carcinoma. Cancer 2006;106:87-94.

5 Risinger JI, Hayes AK, Berchuck A, et al. PTEN/ MMAC1 mutations in endometrial cancers. Cancer Res 1997;57:4736-4738.

6 Kong D, Suzuki A, Zou TT, et al. PTEN1 is frequently mutated in primary endometrial carcinomas. Nat Genet 1997;17:143-144.

7 Zhang S, Yu D. PI(3)king apart PTEN's role in cancer. Clin Cancer Res 2010;16:4325-4330.

8 Djordjevic B, Hennessy BT, Li J, et al. Clinical assessment of PTEN loss in endometrial carcinoma: immunohistochemistry outperforms gene sequencing. Mod Pathol 2012;25:699-708.

9 Mutter GL, Lin MC, Fitzgerald JT, et al. Altered PTEN expression as a diagnostic marker for the earliest endometrial precancers. J Natl Cancer Inst 2000;92: 924-930.

10 Mutter GL, Ince TA, Baak JP, et al. Molecular identification of latent precancers in histologically normal endometrium. Cancer Res 2001;61:4311-4314.

11 Levine RL, Cargile CB, Blazes MS, et al. PTEN mutations and microsatellite instability in complex atypical hyperplasia, a precursor lesion to uterine endometrioid carcinoma. Cancer Res 1998;58: 3254-3258.

12 Jovanovic AS, Boynton KA, Mutter GL. Uteri of women with endometrial carcinoma contain a histopathological spectrum of monoclonal putative precancers, some with microsatellite instability. Cancer Res 1996;56:1917-1921.

13 Cohn DE, Basil JB, Venegoni AR, et al. Absence of PTEN repeat tract mutation in endometrial cancers with microsatellite instability. Gynecol Oncol 2000; 79:101-106.

14 Tashiro H, Blazes MS, Wu R, et al. Mutations in PTEN are frequent in endometrial carcinoma but rare in other common gynecological malignancies. Cancer Res 1997;57:3935-3940.

15 Bussaglia E, del Rio E, Matias-Guiu X, et al. PTEN mutations in endometrial carcinomas: a molecular and clinicopathologic analysis of 38 cases. Hum Pathol 2000;31:312-317.

16 Bilbao C, Rodriguez G, Ramirez R, et al. The relationship between microsatellite instability and PTEN gene mutations in endometrial cancer. Int $\mathrm{J}$ Cancer 2006;119:563-570.

17 Kanaya T, Kyo S, Sakaguchi J, et al. Association of mismatch repair deficiency with PTEN frameshift mutations in endometrial cancers and the precursors in a Japanese population. Am J Clin Pathol 2005; 124:89-96.
18 Peiro G, Lohse P, Mayr D, et al. Insulin-like growth factor-I receptor and PTEN protein expression in endometrial carcinoma. Correlation with bax and bcl2 expression, microsatellite instability status, and outcome. Am J Clin Pathol 2003;120:78-85.

19 Risinger JI, Hayes K, Maxwell GL, et al. PTEN mutation in endometrial cancers is associated with favorable clinical and pathologic characteristics. Clin Cancer Res 1998;4:3005-3010.

20 An HJ, Kim KI, Kim JY, et al. Microsatellite instability in endometrioid type endometrial adenocarcinoma is associated with poor prognostic indicators. Am J Surg Pathol 2007;31:846-853.

21 Rudd ML, Price JC, Fogoros S, et al. A unique spectrum of somatic PIK3CA (p110alpha) mutations within primary endometrial carcinomas. Clin Cancer Res 2011;17:1331-1340.

22 Sun H, Enomoto T, Fujita M, et al. Mutational analysis of the PTEN gene in endometrial carcinoma and hyperplasia. Am J Clin Pathol 2001;115:32-38.

23 An HJ, Logani S, Isacson C, et al. Molecular characterization of uterine clear cell carcinoma. Mod Pathol 2004;17:530-537.

24 Lax SF, Kendall B, Tashiro H, et al. The frequency of p53, K-ras mutations, and microsatellite instability differs in uterine endometrioid and serous carcinoma: evidence of distinct molecular genetic pathways. Cancer 2000;88:814-824.

25 Duggan BD, Felix JC, Muderspach LI, et al. Microsatellite instability in sporadic endometrial carcinoma. J Natl Cancer Inst 1994;86:1216-1221.

26 Caduff RF, Johnston CM, Svoboda-Newman SM, et al. Clinical and pathological significance of microsatellite instability in sporadic endometrial carcinoma. Am J Pathol 1996;148:1671-1678.

27 Aarnio M, Sankila R, Pukkala E, et al. Cancer risk in mutation carriers of DNA-mismatch-repair genes. Int J Cancer 1999;81:214-218.

28 Garg K, Broaddus RR, Soslow RA, et al. Pathologic scoring of PTEN immunohistochemistry in endometrial carcinoma is highly reproducible. Int J Gynecol Pathol 2012;31:48-56.

29 Soslow RA. Endometrial carcinomas with ambiguous features. Semin Diagn Pathol 2010;27:261-273.

30 Garg K, Leitao MM Jr., Kauff ND, et al. Selection of endometrial carcinomas for DNA mismatch repair protein immunohistochemistry using patient age and tumor morphology enhances detection of mismatch repair abnormalities. Am J Surg Pathol 2009;33:925-933.

31 Zhou XP, Kuismanen S, Nystrom-Lahti M, et al. Distinct PTEN mutational spectra in hereditary non-polyposis colon cancer syndrome-related endometrial carcinomas compared to sporadic microsatellite unstable tumors. Hum Mol Genet 2002;11: $445-450$

32 Djordjevic B, Hennessy BT, Li J, et al. Clinical assessment of PTEN loss in endometrial carcinoma: immunohistochemistry out-performs gene sequencing. Mod Pathol 2012;25:699-708.

33 Cheung LW, Hennessy BT, Li J, et al. High frequency of PIK3R1 and PIK3R2 mutations in endometrial cancer elucidates a novel mechanism for regulation of PTEN protein stability. Cancer Discov 2011;1:170-185.

34 Catasus L, Gallardo A, Cuatrecasas M, et al. Concomitant PI3K-AKT and p53 alterations in endometrial carcinomas are associated with poor prognosis. Mod Pathol 2009;22:522-529. 
35 Garg K, Shih K, Barakat R, et al. Endometrial carcinomas in women aged 40 years and younger: tumors associated with loss of DNA mismatch repair proteins comprise a distinct clinicopathologic subset. Am J Surg Pathol 2009;33:1869-1877.

36 Alkushi A, Clarke BA, Akbari M, et al. Identification of prognostically relevant and reproducible subsets of endometrial adenocarcinoma based on clustering analysis of immunostaining data. Mod Pathol 2007; 20:1156-1165.

37 McConechy MK, Ding J, Cheang MC, et al. Use of mutation profiles to refine the classification of endometrial carcinomas. J Pathol 2012;228:20-30.

38 Catasus L, D’Angelo E, Pons C, et al. Expression profiling of 22 genes involved in the PI3K-AKT pathway identifies two subgroups of high-grade endometrial carcinomas with different molecular alterations. Mod Pathol 2010;23:694-702.

39 Prat A, Ellis MJ, Perou CM. Practical implications of gene-expression-based assays for breast oncologists. Nat Rev Clin Oncol 2012;9:48-57.

40 Carden CP, Yap TA, Kaye SB. PARP inhibition: targeting the Achilles' heel of DNA repair to treat germline and sporadic ovarian cancers. Curr Opin Oncol 2010;22:473-480.

41 Umar A, Boland CR, Terdiman JP, et al. Revised Bethesda Guidelines for hereditary nonpolyposis colorectal cancer (Lynch syndrome) and microsatellite instability. J Natl Cancer Inst 2004;96:261-268.

42 Lancaster JM, Powell CB, Kauff ND, et al. Society of Gynecologic Oncologists Education Committee statement on risk assessment for inherited gynecologic cancer predispositions. Gynecol Oncol 2007;107:159-162.

43 Westin SN, Lacour RA, Urbauer DL, et al. Carcinoma of the lower uterine segment: a newly described association with Lynch syndrome. J Clin Oncol 2008;26: 5965-5971.

44 Garg K, Soslow RA. Lynch syndrome (hereditary nonpolyposis colorectal cancer) and endometrial carcinoma. J Clin Pathol 2009;62:679-684.

45 Berends MJ, Wu Y, Sijmons RH, et al. Molecular and clinical characteristics of MSH6 variants: an analysis of 25 index carriers of a germline variant. Am J Hum Genet 2002;70:26-37.

46 Mercado RC, Hampel H, Kastrinos F, et al. Performance of PREMM $(1,2,6)$, MMRpredict, and MMRpro in detecting Lynch syndrome among endometrial cancer cases. Genet Med 2012;14:670-680.

47 Ladabaum U, Wang G, Terdiman J, et al. Strategies to identify the Lynch syndrome among patients with colorectal cancer: a cost-effectiveness analysis. Ann Intern Med 2011;155:69-79.
48 Resnick K, Straughn JM Jr., Backes F, et al. Lynch syndrome screening strategies among newly diagnosed endometrial cancer patients. Obstet Gynecol 2009;114: 530-536.

49 Bartley AN, Luthra R, Saraiya DS, et al. Identification of cancer patients with Lynch syndrome: clinically significant discordances and problems in tissue-based mismatch repair testing. Cancer Prev Res (Phila) 2012; 5:320-327.

50 Baudhuin LM, Burgart LJ, Leontovich O, et al. Use of microsatellite instability and immunohistochemistry testing for the identification of individuals at risk for Lynch syndrome. Fam Cancer 2005;4:255-265.

51 Kwon JS, Scott JL, Gilks CB, et al. Testing women with endometrial cancer to detect Lynch syndrome. J Clin Oncol 2011;29:2247-2252.

52 Bellcross CA, Bedrosian SR, Daniels E, et al. Implementing screening for Lynch syndrome among patients with newly diagnosed colorectal cancer: summary of a public health/clinical collaborative meeting. Genet Med 2012;14:152-162.

53 Kalloger SE, Allo G, Mulligan AM, et al. Use of mismatch repair immunohistochemistry and microsatellite instability testing: exploring Canadian practices. Am J Surg Pathol 2012;36:560-569.

54 Deng G, Nguyen A, Tanaka H, et al. Regional hypermethylation and global hypomethylation are associated with altered chromatin conformation and histone acetylation in colorectal cancer. Int J Cancer 2006;118:2999-3005.

55 Domingo E, Laiho $\mathrm{P}$, Ollikainen $\mathrm{M}$, et al. BRAF screening as a low-cost effective strategy for simplifying HNPCC genetic testing. J Med Genet 2004;41:664-668.

56 Loughrey MB, Waring PM, Tan A, et al. Incorporation of somatic BRAF mutation testing into an algorithm for the investigation of hereditary non-polyposis colorectal cancer. Fam Cancer 2007;6:301-310.

57 McGivern A, Wynter CV, Whitehall VL, et al. Promoter hypermethylation frequency and BRAF mutations distinguish hereditary non-polyposis colon cancer from sporadic MSI-H colon cancer. Fam Cancer 2004; 3:101-107.

58 Peterson LM, Kipp BR, Halling KC, et al. Molecular characterization of endometrial cancer: a correlative study assessing microsatellite instability, MLH1 hypermethylation, DNA mismatch repair protein expression, and PTEN, PIK3CA, KRAS, and BRAF mutation analysis. Int J Gynecol Pathol 2012;31:195-205.

59 Mutch DG, Powell MA, Mallon MA, et al. RAS/RAF mutation and defective DNA mismatch repair in endometrial cancers. Am J Obstet Gynecol 2004; 190:935-942. 